\title{
Intervención Psicológica para la Gestión del Estrés en Deportistas Juveniles Federados
}

\section{Psychological Intervention for Stress Management in Federated Youth Athletes}

Valeris Pérez Esquivel. ${ }^{1}$, Yanet Díaz Martín. ${ }^{2}$

Recibido: 10-02-2019 / Revisado: 15-02-209 /Aceptado: 04-03-2019/ Publicado: 14-06-2019

\begin{abstract}
. https://doi.org/10.33262/cienciadigital.v3i2.5.535

The study consisted in the elaboration of a quasi-experiment, to verify the effectiveness of a system of intervention techniques, applied to a non-probability sample of 16 young athletes belonging to the Chimborazo Sports Federation, sportsmen between 17 and 18 years old, whose intervention was during a period of five months. The intervention takes as its theoretical focus the transactional theory of stress, incorporating techniques of psychoeducation, cognitive restructuring, emotional management, control of physiological responses and management of environmental demands. The main instruments used were: the stress assessment scale in the sports environment, the 20 elements related to stress management from the Performance-related Psychological Characteristics Test and an interview that explores stress experiences in competitive situations. The data obtained with the statistical tools were processed by applying the frequency distribution, percentage calculation, arithmetic mean and statistical significance test for serial pairs. The results show significant differences between the experimental group and the control group, before and after the application of the intervention. The development and systematization of stress management techniques
\end{abstract}

\footnotetext{
${ }^{1}$ Federación Deportiva de Chimborazo, Riobamba, Ecuador. fedechimborazo24@yahoo.es

2 Universidad Técnica de Ambato, Facultad de Ciencias de la Salud. Ambato, Ecuador. ya.diaz@uta.edu.ec
} 
adapted to the demands of sport from a transactional perspective is presented as a scientific novelty. It is concluded as a practical value the development of an information-gathering sheet that allows capturing the resources trained in the athlete to integrate them in mental preparation routines and use them in potentially stressful situations.

Key Words: Stress, Transactional, Techniques, Athletes.

\section{Resumen.}

El estudio consistió en la elaboración de un cuasiexperimento, para verificar la efectividad de un sistema de técnicas de intervención, aplicado a una muestra no probabilística de 16 atletas jóvenes pertenecientes a la Federación Deportiva Chimborazo, deportistas entre 17 y 18 años, cuya intervención fue durante un período de cinco meses. La intervención toma como foco teórico la Teoría transaccional del estrés, incorporando técnicas de psicoeducación, reestructuración cognitiva, manejo emocional, control de respuestas fisiológicas y manejo de demandas ambientales. Se utilizaron como instrumentos principales: la escala de evaluación del estrés en el entorno deportivo, los 20 elementos relacionados con el manejo del estrés de la Prueba de características psicológicas relacionadas con el rendimiento y una entrevista que explora las experiencias de estrés en situaciones competitivas. Los datos obtenidos con las herramientas estadísticas se procesaron aplicando la distribución de frecuencia, el cálculo del porcentaje, la media aritmética y la prueba de significación estadística para pares en serie. Los resultados muestran diferencias significativas entre el grupo experimental y el grupo control, antes y después de la aplicación de la intervención. El desarrollo y sistematización de técnicas de manejo del estrés adaptadas a las demandas del deporte desde una perspectiva transaccional, se presenta como una novedad científica. Se concluye como un valor práctico el desarrollo de una hoja de recopilación de información que permite capturar los recursos entrenados en el deportista para integrarlos en rutinas de preparación mental y usarlos en situaciones potencialmente estresantes.

Palabras Claves: Estrés, Transaccional, Técnicas, Deportistas.

\section{Introducción}

La Categoría "Estrés" ha sido ampliamente tratada desde la Psicología Clínica, donde puede referenciarse que el pionero en sus estudios, resultó ser el médico fisiólogo Austriaco Hans Selye en 1930, el que, sobre la base de las investigaciones realizadas en la materia en los 
años 36 y 50 del pasado siglo menciona en sus estudio sobre el estrés (García, 2011), posteriormente devino además el precursor de la teoría y la metodología de la doble periodización del entrenamiento deportivo, al aportar importantes supuestos desde las reacciones fisiológicas del organismo a las demandas del entorno.

Años más tarde Bandura (1983), como principal exponente de la escuela psicológica de aprendizaje social, consideró la personalidad cómo una interacción entre el ambiente, los procesos psicológicos del sujeto y la conducta, demostrando la necesidad de integrar en el estudio del desarrollo del individuo de forma dinámica las condicionantes tanto biológicas, cómo psicológicas y ambientales, las cuales le hagan al sujeto más vulnerable o más resistente a las situaciones de riesgo (Moreno-Jiménez, 2005). La comprobación experimental de la proposición expresada con anterioridad, así como su vigencia tanto en la explicación de los procesos de socialización, como en el marco clínico, permitió evaluar de forma más dinámica y multifactorial la aparición del estrés en el individuo. De tal manera que Folkman y Lazarus (1986), lo definen cómo el resultado de la relación entre el individuo y el entorno, siendo evaluado por aquel como amenazante, que desbordan sus recursos y pone en peligro su bienestar.

En el presente siglo Andrade, Lois y Arce (2007), lo conceptualizan como presión del medio ambiente externo o interno, que impone demandas físicas y o emocionales que requieren reajustes tanto a nivel fisiológicos como psicológicos por parte del individuo. Resultando esta última definición la rectora de la presente investigación, ateniendo a la necesidad de que el estrés sea tratado de manera integradora desde las condicionantes: biológicas, psicológicas y sociales de la personalidad.

El tratamiento a los estados desfavorables que presenta el deportista antes de la competición posee varias aristas, pues ha sido valorado como pre arranque, ansiedad competitiva y estrés, siendo este último el que por sus avances en la concepción teórica, permite desde el punto vista metodológico brindar un tratamiento más integral al atleta, convirtiendo su afrontamiento en un entrenamiento mental, que garantiza la reestructuración de pensamientos negativos, la gestión de emociones y síntomas fisiológicos, así como el manejo del entorno; en cualquier caso, el estado de estrés-recuperación del sujeto será el indicador que nos permitirá conocer el nivel de estrés físico y mental del individuo, para utilizar estrategias en su recuperación (Molinero, 2012), requiriendo la práctica de las técnicas a utilizar desde el inicio de la preparación deportiva.

En materia de intervenciones realizadas con deportistas para la gestión el estrés, destaca el estudio de Piqueras y Casinelo (2011), los que proponen diferentes técnicas para el afrontamiento a la ansiedad y el estrés, partiendo de evaluar sus manifestaciones en las esferas cognitiva, emocional, fisiológica, conductual, así como las consecuencias que trae al deportista. 
Atendiendo a estudios de tipo descriptivo Gonzales, Valdivia Zagales y Romero (2015), tras la revisión de siete estudios realizados por distintos autores, en los que en su mayoría aplicaron el Test de Características Psicológicas Relacionadas al Rendimiento (CPRD) el cual fue validado al castellano por Gimeno (1998) basándose en un instrumento americano denominado: Psychological Skills Inventory for Sports (PSIS) el cual adapta los estándares del análisis psicológico de las características del ser humano, aplicándolo al ámbito deportivo (Cabal, 2018) existiendo un consenso en evaluar la autoconfianza como un factor importante para el control del estrés, correlacionando inversamente la presencia de elevados niveles de confianza con la aparición de ansiedad y estrés en el deportista.

Retomando con mayor actualidad, las intervenciones sobre el tema tratado en deportistas, destaca la investigación de Suntaxi (2015), quien, partiendo de un estudio descriptivo sobre la presencia de estrés en atletas de Taekwondo, propone un manual de técnicas de relajación para disminuir el nivel del mismo y aumentar el rendimiento deportivo, desarrollando fundamentalmente la intervención sobre los síntomas fisiológicos que provoca el estrés.

El mismo año aparece la tesis de grado de Garzón (2015), que muestra también de forma integral el manejo de estados de pre arranque en el deportista, haciendo referencia a su tratamiento como situaciones de estrés y plasmando técnicas para la gestión del mismo desde lo fisiológico, cognitivo y relación con el entorno, destacando en su generalidad técnicas clásicas utilizadas por la terapia racional emotiva conductual.

Por su parte Almeida (2017), realiza un estudio comparativo de los niveles subjetivos de estrés-recuperación entre deportistas pertenecientes a las agrupaciones de deportes individuales y colectivos, aun cuando en la muestra estudiada no se encontraron valores estadísticos significativos relacionados con la presencia de estrés, si se manifestaron puntuaciones más altas en atletas de deportes individuales.

En el acercamiento inicial a un grupo de 16 deportistas, priorizados para atención individualizada, con vistas a su participación en los Juegos Nacionales Juveniles del año 2018, pudo constatarse que los mismos manifestaron dificultades en la gestión del Estrés principalmente en situaciones de aumento considerable de las cargas de entrenamiento, así como en el entorno competitivo.

Por lo que el presente estudio aborda como situación problémica: Las insuficiencias detectadas en la preparación psicológica de los deportistas pre seleccionados para los Juegos Nacionales Juveniles de 2018, relacionadas con la habilidad para gestionar el estrés, por consiguiente, el objetivo fue comprobar la efectividad de un sistema de técnicas para desarrollar la habilidad de gestionar el estrés en deportistas juvenil.

\section{Pregunta Cientifica}


¿El sistema de intervención psicológica propuesto, resultará efectivo para desarrollar la habilidad de gestionar el estrés en deportistas juveniles de la Federación Deportiva de Chimborazo?

\section{Tareas de investigación:}

Revisión de la literatura especializada relacionada con la gestión del estrés desde el modelo transaccional y el desarrollo de la habilidad de gestionar el estrés en el contexto deportivo.

Diagnóstico inicial de la habilidad de gestionar el estrés en deportistas juveniles de Federación Deportiva de Chimborazo, pre-seleccionados para los juegos Nacionales Juveniles 2018.

Diseño e implementación de un sistema de técnicas para el desarrollo de la habilidad de gestionar el estrés en deportistas juveniles.

Comprobación de la efectividad del sistema de técnicas propuesto mediante la elaboración de un diseño experimental de tipo cuasiexperimento.

\section{Metodología}

Para cumplir con los objetivos propuestos, se realizó un estudio de tipo cuasiexperimental; de acuerdo con Mocha Bonilla (2018) quien afirma que un programa de intervención debe estar basado en un grupo experimental y en un grupo control, planteado la selección de los sujetos y la implementación de un programa de intervención con etapas bien definidas: adaptación, estabilización, mantenimiento y evaluación, siempre tomando en cuenta las características psicológicas y físicas de los sujetos de estudio, en función de la edad y la condición física. Por consiguiente, se aplicó un enfoque mixto con una intervención antes y después (pre y post test).

El estudio fue desarrollado en el periodo de febrero a junio del año 2018, con una muestra no probabilística o intencional de 16 deportistas juveniles 6 damas y 10 varones con edades comprendidas entre los 17 y 18 años, pertenecientes a la Federación Deportiva de Chimborazo de la ciudad de Riobamba, Ecuador, pre seleccionados para los juegos nacionales Juveniles de 2018, todos pertenecientes a deportes individuales, los que se dividieron en dos grupos de ocho: (3 damas y 5 varones respectivamente).

Tanto al grupo experimental como al de control, se les aplicó antes y después de la intervención, la escala de valoración del estrés en el ámbito deportivo, los 20 ítems vinculados con la gestión del estrés del Test de Características Psicológicas Relacionadas al Rendimiento (CPRD) aportado por Gimeno (2010) citado en (Paz-Llanos, 2019), así como una entrevista que aborda las vivencias de estrés en situaciones competitivas. 
Se estableció una comparación entre el grupo experimental y el grupo de control, antes y después de la intervención, entre la cantidad de deportistas que experimentaron mejorías en la Escala de Valoración del Estrés en el Ámbito Deportivo, elaborada por Pedrosa, SuarezÁlvarez y García-Cueto (2012), así como los que progresaron en la ubicación en el percentil relacionado con las 20 proposiciones del Test de Características Psicológicas Vinculadas al Rendimiento Deportivo (CPRD).

Ambos test al presentar un formato de respuesta de escala Likert numérica, permitieron procesar los datos con la prueba de significación estadística para pares seriados, con un índice de significación $\mathrm{p}<0.05$, con la utilización del paquete estadístico SPSS versión 24.0

Resultó de gran utilidad, dado el estrecho vínculo que guarda la autoconfianza del deportista con la gestión del estrés, establecer una comparación con algunos de los ítems seleccionados del Test de Características Psicológicas Relacionadas al Rendimiento, que abordan las variables de la confianza que posee el atleta en sus propias capacidades y habilidades para enfrentar los retos deportivos, expresado en la creencia de logro de elevados desempeños.

Se compararon además en momentos antes y después de la intervención, las respuestas ofrecidas en la entrevista semi estructurada, la que contó con un total de 10 preguntas, encaminadas a la obtención de datos referentes a la gestión del estrés por parte del deportista en situaciones competitivas, procesando las respuestas obtenidas en el Excel 2016, que permitió comparar los datos con la confección de gráficos circulares y de barras.

La variable de investigación fue el estrés visto como la presión del medio ambiente externo o interno que impone demandas físicas y o emocionales que requieren reajustes tanto fisiológicos como psicológicos por parte del individuo. Lo que conllevó a identificar indicadores en la variable de estudio distribuidos, en las diferentes esferas de la personalidad en las que se expresa el estrés.

Esfera cognitiva: Aparición de confusión, presencia persistente de recuerdos negativos de experiencias anteriores $\mathrm{u}$ olvido frecuentes, presencia de cogniciones negativas, preocupaciones en relación a las capacidades para afrontar el reto de la competición, confianza en las habilidades para afrontar los retos deportivos.

Esfera emocional: Presencia de sentimientos de frustración, temores persistentes a los contrarios o a la situación competitiva, incidencia de mal humor o cambios frecuentes del mismo.

Esfera fisiológica: Incidencia de elevados niveles de tensión física, temblores, pérdida del apetito, dificultades para conciliar el sueño, sudoraciones, palpitaciones.

\section{Variables de control}

Edad cronológica: Años de edad que posee el deportista. 
Edad Deportiva: Tiempo de permanencia del atleta en la práctica del deporte.

Autovaloración adecuada: Correspondencia entre lo que piensa el atleta de sí mismo y lo que consideran los demás en relación al rendimiento deportivo.

Adecuado estado de salud: Ausencia de enfermedades o lesiones deportivas.

La intervención fue realizada en un total de 18 sesiones de trabajo, las que preferentemente se distribuyeron con una frecuencia de una vez por semana, estando integrada por 14 técnicas pertenecientes fundamentalmente al modelo de la Terapia Cognitiva Conductual, de donde también procede el enfoque transaccional del estrés de Lazarus y Folkman, por lo que se utilizó terapias cognitivo-conductuales orientadas al tratamiento de la depresión en población adolescente, en función de los factores cognitivos (Mateos-Pérez, 2019).

Reestructuración cognitiva o manejo de pensamientos negativos e ideas irracionales, la gestión emocional que estuvo dirigida a la identificación y modulación de emociones tanto negativas como positivas, el tratamiento de síntomas fisiológicos provocados por la vivencia del estrés, así como el manejo del entorno.

Las técnicas referidas con anterioridad estuvieron apoyadas por el Protocolo de Recogida de datos, que consistió en una planilla en la que se plasma de forma integral, los elementos referentes a las situaciones que potencian en el deportista la aparición del estrés desde todas las dimensiones, incluyendo las exigencias del entorno, la percepción que se tienen de estas exigencias y las respuestas emocionales y fisiológicas que provocan.

Al lado de cada situación potenciadora del estrés, se colocaron las técnicas de intervención encaminadas a la gestión del mismo, lo que permitió además una retroalimentación en lo referido a cuál de las técnicas fueron las más utilizadas, dentro de ellas las más efectivas, e incluso poder reestructurar aquellas que no tuvieron en el atleta el efecto deseado. Algunas de las técnicas utilizadas se presentan en la siguiente tabla:

Tabla 1. Ejemplos de técnicas de gestión de estrés por esferas.

$\begin{array}{lll}\text { Restructuración } & \text { Gestión Emocional } & \begin{array}{l}\text { Gestión de Síntomas } \\ \text { fisiológicos }\end{array} \\ \text { Cognitiva } & & \text { Manejo del entorno }\end{array}$

\begin{tabular}{l}
\hline Técnica 3 Corazón \\
triste corazón feliz \\
Materiales: \\
Hoja de papel en \\
blanco, lápices de \\
colores, \\
esferográfico. \\
Objetivo: \\
Desarrollar en el \\
atleta la habilidad de \\
identificar
\end{tabular}

Técnica 6 Caricatura de mis contrarios Materiales:

Hoja de papel en blanco, lápices de colores.

Objetivos:

Facilitar en el atleta la gestión de emociones negativas en relación con la presencia de

Técnica 8
Acondicionamiento
físico
Materiales:
Implementos deportivos
en los deportes que los
utilicen.
Objetivos:
Realizar el calentamiento
acorde al nivel de

Técnica 12

Similar a mi escenario

Materiales:

Ninguno

Objetivos:

Facilitar la adaptación del

deportista a nuevos

escenarios competitivos. 
pensamientos determinados

positivos y negativos contrarios en la

en el deporte.

contrarios en
competición. activación fisiológica que posea el deportista.

Fuente: Elaboración propia.

Es válido manifestar que aun cuando se exponen las técnicas de forma individualizada y divididas por cada dimensión, al final se integran en lo que constituye un ritual que permite al deportista la gestión del estrés en aquellas situaciones que potencien su aparición, fundamentalmente en periodos de entrenamiento de grandes cargas físicas y en la competencia deportiva.

\section{Resultados}

El procesamiento estadístico de los datos aportados por las diferentes técnicas aplicadas, permitió obtener los siguientes resultados:

En la aplicación de la prueba de significación estadística para pares seriados, tanto en la Escala de Valoración del Estrés, como en los ítems seleccionados del Test de Características Psicológicas Relacionadas al Rendimiento Deportivo (CPRD), se apreciaron resultados con índices de significación $p<0.05$ en el grupo experimental (ver tabla 2), en tanto en el grupo control los valores fueron mayores a dicha magnitud.

Tabla 2. Resultados Prueba de significación estadística

\begin{tabular}{lll} 
Test aplicado & Grupos de estudio & Significación pre y post intervención \\
\hline E. de Valoración de Estrés & Experimental & $\mathbf{0 . 0 5}$ \\
E. de Valoración de Estrés & Control & 0.50 \\
CPRD & Experimental & $\mathbf{0 . 0 3}$ \\
CPRD & Control & 1.77
\end{tabular}

Fuente: Elaboración propia.

Puede observarse en los datos tabulados con anterioridad, la diferencia entre los niveles de significación entre los grupos estudiados, en los que fundamentalmente los mayores contrastes se establecieron en el Test de Características Psicológicas Relacionadas con el Rendimiento Deportivo, permitiendo inferir que los cambios obtenidos en materia del desarrollo de la habilidad de gestionar el estrés no fueron producto del azahar o de la actuación de otras variables ajenas al estudio.

Estableciendo la escala Likert desde 0 hasta 4 puntos para ambos test, brindando las menores magnitudes a las respuestas que indicaran dificultades en la habilidad de gestionar el estrés, se pudieron comparar los valores totales obtenidos para ambas pruebas antes y después de la 
aplicación del conjunto de técnicas de intervención tanto en el grupo experimental como en el de control.

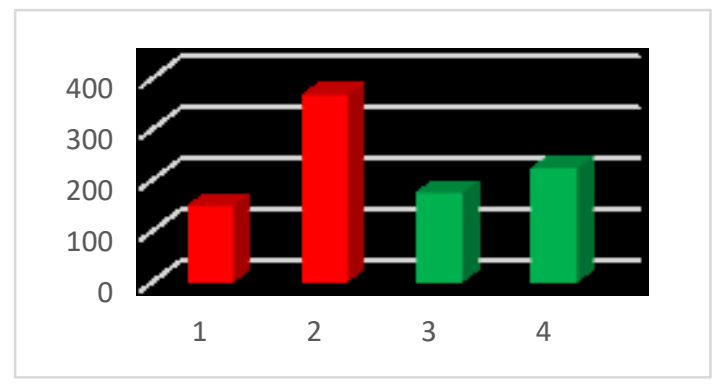

Grupo experimental Grupo Control

Figura 1. Comparación Escala de Estrés Fuente: Elaboración propia.

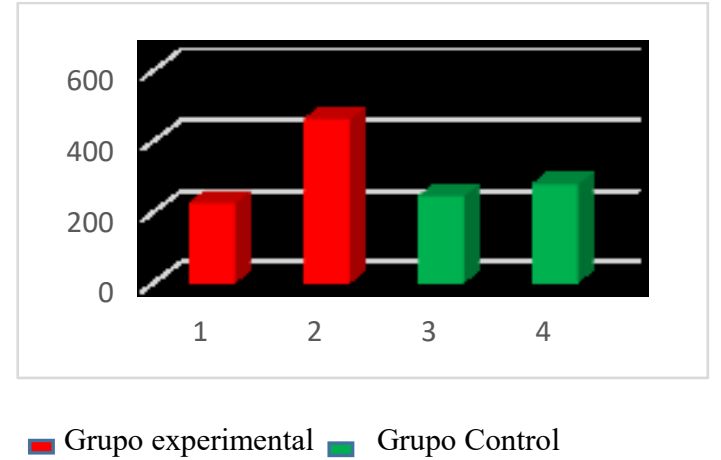

Figura 2. Comparación CPRD

Fuente: Elaboración Propia.

Puede apreciarse, como en el diagnóstico inicial del desarrollo de la habilidad de gestionar el estrés estudiado en ambos grupos, se observaron totales de puntuaciones muy bajas, apuntando a las insuficiencias en la gestión del estrés en los deportistas juveniles de Federación Deportiva de Chimborazo, pre-seleccionados para participar en los Juegos Nacionales Juveniles de 2018.

Los datos arrojados por el procesamiento estadístico, permiten establecer una comparación con el estudio propuesto por Almeida (2017), en el que la investigadora no encontró elementos significativos que denotaran insuficiencias en el manejo de estrés en los deportistas estudiados, aun en una muestra de mayor tamaño, sin embargo puede evaluarse le necesidad de valorar el factor edad y experiencia deportiva, si se tiene en cuenta que en el mencionado estudio, se accedió a una muestra con atletas que promediaban 25 años de edad.

En la Escala de Estrés Percibido en el Ámbito Deportivo, se expresó en el grupo experimental una diferencia de 217 puntos entre los obtenidos antes y después de la aplicación del sistema de intervención a favor de las mejoras en la habilidad para gestionar el estrés, en tanto en el grupo control fue solo de 48 puntos en condiciones pre y post intervención.

Resultado similar se expresó en los ítems seleccionados del Test de Características Psicológicas Relacionadas al Rendimiento Deportivo, donde en el grupo experimental se obtuvo una diferencia pre y post tratamiento de 223 puntos, en tanto en el grupo control solo alcanzó la cifra de 44 puntos.

El resultado obtenido en ambos test, se ilustra en las tablas 3 y 4 expuestas a continuación, que reflejan tanto en el grupo experimental (GE) como en el de control (GC), el porciento de estudiantes que se ubicaban en los diferentes rangos percentiles antes y después de la intervención, observándose las diferencias en puntos porcentuales en ambos grupos. 
Tabla 3. Porciento de atletas ubicados por rango en la Escala de Percepción de Estrés pre y post intervención
Tabla 4 Porciento de atletas ubicados por rango en los ítems seleccionados del test CPRD

\begin{tabular}{lllll} 
Rangos & $\begin{array}{l}\text { G E } \\
\text { Antes }\end{array}$ & $\begin{array}{l}\text { GE } \\
\text { Después }\end{array}$ & $\begin{array}{l}\text { GC } \\
\text { Antes }\end{array}$ & $\begin{array}{l}\text { GC } \\
\text { Después }\end{array}$ \\
\hline $00-12$ & $25 \%$ & $0 \%$ & $0 \%$ & $0 \%$ \\
$13-24$ & $50 \%$ & $0 \%$ & $62.5 \%$ & $50 \%$ \\
$25-36$ & $25 \%$ & $25 \%$ & $37.5 \%$ & $50 \%$ \\
$37-48$ & $0 \%$ & $50 \%$ & $0 \%$ & $0 \%$ \\
$49-60$ & $0 \%$ & $25 \%$ & $0 \%$ & $0 \%$
\end{tabular}

Fuente: Elaboración propia.

\begin{tabular}{lllll} 
Rangos & $\begin{array}{l}\text { G E } \\
\text { Antes }\end{array}$ & $\begin{array}{l}\text { GE } \\
\text { Después }\end{array}$ & $\begin{array}{l}\text { GC } \\
\text { Antes }\end{array}$ & $\begin{array}{l}\text { GC } \\
\text { Después }\end{array}$ \\
\hline $00-16$ & $0 \%$ & $0 \%$ & $0 \%$ & $0 \%$ \\
$17-32$ & $75 \%$ & $0 \%$ & $62.5 \%$ & $37.5 \%$ \\
$33-48$ & $25 \%$ & $37.5 \%$ & $37.5 \%$ & $62.5 \%$ \\
$49-64$ & $0 \%$ & $25 \%$ & $0 \%$ & $0 \%$ \\
$65-80$ & $0 \%$ & $37.5 \%$ & $0 \%$ & $0 \%$
\end{tabular}

Fuente: Elaboración propia.

La entrevista permitió comparar las mejoras en las respuestas ofrecidas por ambos grupos pre y post intervención, con la utilización del cálculo porcentual (ver gráfico 3), en la que se obtuvo una mejora de apenas un $17 \%$ en el grupo de control representando un total de 8 respuestas, en tanto el grupo experimental expresó un $83 \%$ de mejoras en las respuestas encaminadas al afrontamiento del estrés, lo que representó un total de 40 respuestas.

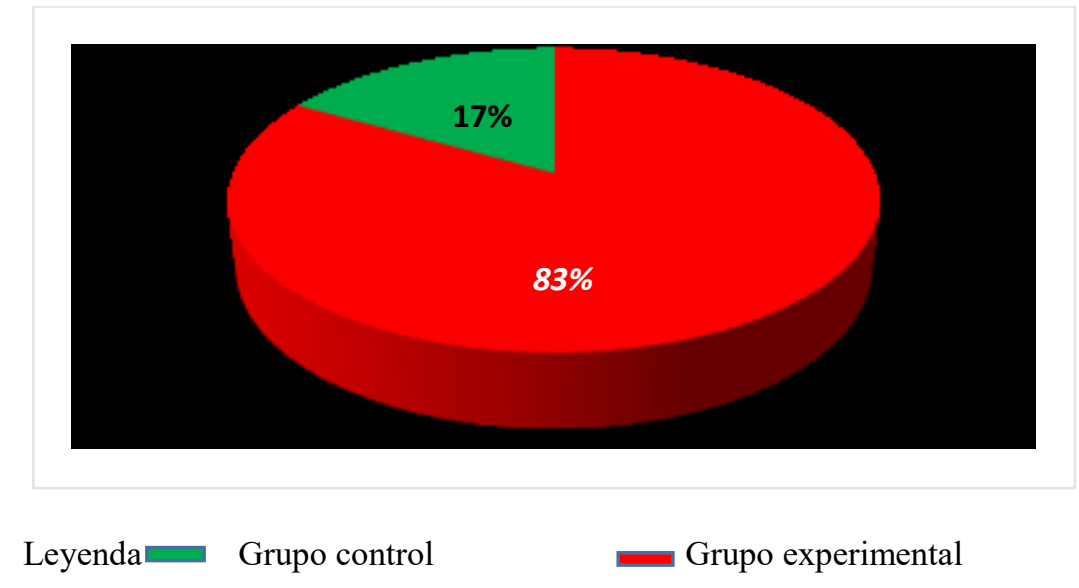

Figura 3. Comparación en las mejoras de respuestas a la entrevista entre ambos grupos.

Fuente: Elaboración propia.

Dentro de los 20 ítems que se encaminan a diagnosticar el control del estrés, en el Test de Características Psicológicas Relacionadas al Rendimiento, aparecen 10 proposiciones que evalúan en el deportista la confianza que posee en sus propias fuerzas, permitiendo al atleta reflejar la valoración que el mismo realiza de su preparación, la proyección hacia futuros desempeños en torno a la competición, así como la fluctuación que puede tener su confianza al aparecer lesiones u obstáculos durante la actividad competitiva. 
La siguiente tabla, muestra las diferencias de medias aritméticas obtenidas en cinco de estas preguntas, las que aparecen enunciadas en el test de forma positiva, permitiendo contrastar el grupo experimental y de control antes y después de la intervención, teniendo en cuenta que medias más elevadas indican un mayor desarrollo de la habilidad de gestionar el estrés:

Tabla 5. Comparación de medias aritméticas del test CPRD

\begin{tabular}{lllll} 
Preguntas & $\begin{array}{l}\text { Media GE } \\
\text { Antes }\end{array}$ & $\begin{array}{l}\text { Media GE } \\
\text { Después }\end{array}$ & $\begin{array}{l}\text { Media GC } \\
\text { Antes }\end{array}$ & $\begin{array}{l}\text { Media GC } \\
\text { Después }\end{array}$ \\
\hline Tengo gran confianza en mí técnica & 0.75 & 3.00 & 1.87 & 2.00 \\
$\begin{array}{l}\text { En la mayoría de competiciones (partidos), confío } \\
\text { en que lo haré bien }\end{array}$ & 1.00 & 2.64 & 1.75 & 1.75 \\
$\begin{array}{l}\text { Generalmente puedo seguir participando (jugando) } \\
\text { con confianza, aunque se trate de una de mis peores } \\
\text { actuaciones }\end{array}$ & 0.75 & 2.50 & 0.87 & 1.00 \\
$\begin{array}{l}\text { Tengo fe en mí mismo(a) } \\
\begin{array}{l}\text { Suelo confiar en mí mismo(a), aun en los } \\
\text { momentos más difíciles de una competición (o }\end{array} \\
\text { partido) }\end{array} \quad 1.75$ & 3.25 & 1.75 & 1.75 \\
\end{tabular}

Fuente: Elaboración propia.

Los resultados obtenidos, al evaluar las diferencias de las medias aritméticas pre y post intervención entre ambos grupos, en cuanto a las preguntas del Test de Características Psicológicas Relacionadas al Rendimiento vinculadas a la autoconfianza, permiten encontrar puntos coincidentes con el estudio realizado por Gonzales, Valdivia Zagales y Romero (2015), los que luego de una revisión de la literatura concluyen en la existencia de consenso en evaluar la autoconfianza como un factor que se relaciona inversamente con la aparición del estrés.

Integrando los resultados obtenidos en todos los test, puede manifestarse que estos permitieron corroborar la efectividad del sistema de técnicas aplicadas para gestionar el estrés, desde una perspectiva transaccional, expresándose tanto en la Escala de Estrés en el Ámbito Deportivo, como en los ítems seleccionados del test de Características Psicológicas Relacionadas al Rendimiento, marcadas diferencias entre los avances del grupo experimental y de control antes y después del sistema de intervención propuesto.

En efecto los miembros del grupo experimental, lograron alcanzar en un $75 \%$ y $62.5 \%$ los máximos rangos percentiles en ambos test, lo que indican cualitativamente el ser evaluados entre bien y muy bien, mientras en el grupo de control ningún atleta alcanzó estas evaluaciones.

Se manifestaron en los deportistas del grupo experimental, mejorías considerables en el afrontamiento al estrés, en tanto se expresó después de la intervención que los atletas de este 
grupo, comenzaron a percibir como menos amenazantes las exigencias del entorno alrededor de la competición, utilizando en mayor medida las técnicas de reestructuración cognitiva propuestas en la intervención, datos que se pudieron corroborar en las respuestas ofrecidas en la entrevista.

Los deportistas del grupo experimental manifestaron aumentos en la gestión emocional, demostrando menor incidencia de temores, sentimientos de frustración en relación a actuaciones deportivas, y un decrecimiento de los estados de mal humor o cambios constante de estos. Fue notable la disminución de síntomas fisiológicos, como la pérdida del apetito, alteración de la calidad del sueño y tensión física, lo que se corroboró en las diferencias de puntuaciones en la respuesta a ambos test antes y después de la intervención.

\section{Conclusiones}

La bibliografía consultada sobre la gestión del estrés, refleja la importancia de desarrollar la habilidad de gestionar el estrés en el deportista, encontrándose varias técnicas para el afrontamiento del mismo desde las distintas esferas de la personalidad, las que resultan ser en su mayoría clásicas y muy generales, expresándose la necesidad de que se ajusten más a las exigencias del deporte.

El diagnóstico inicial de la habilidad para gestionar el estrés en los deportistas , denotó insuficiencias en su desarrollo, expresado en que el 100\% de la muestra se ubicaba en los rangos más bajos de puntuaciones en los test aplicados, reflejándose en una alta presencia de atletas que percibían las exigencias del entorno de forma amenazante, un predominio de emociones negativas alrededor de la competición, así como la incidencia de múltiples síntomas fisiológicos que afectaban al deportista.

El sistema de intervención, integró un total de 14 técnicas psicológicas aplicadas en 18 sesiones con una frecuencia semanal, encaminadas a la reestructuración cognitiva, la gestión de emociones y el manejo de síntomas fisiológicos del estrés, aportando un conjunto de técnicas que se adaptan a las exigencias del deporte, así como una planilla de gestión de estrés que permite al deportista plasmar todas las técnicas a utilizar por cada esfera e integrarlas en un ritual de competición.

La intervención para desarrollar la habilidad de gestionar el estrés en atletas juveniles de Federación Deportiva de Chimborazo, resultó ser efectiva, demostrándose en las diferencias pre y post tratamientos expresadas entre ambos grupos, donde los integrantes del grupo experimental lograron alcanzar entre un 75\% y 62.5\% los rangos más altos de puntuación en los test aplicados y el grupo de control expresó un $0 \%$, reflejándose marcadas mejorías en el grupo experimental relacionadas con el desarrollo de la habilidad para gestionar el estrés. 


\section{Referencias}

Almeida, E. (2017). Análisis Comparativo de los Niveles Subjetivos de Estrés -Recuperación Entre Deportistas Practicantes de Deportes Individuales y Colectivos. Tesis Aspirante a Especialista en medicina deportiva.

Andrade, E.M., Lois G. y Arce, C. (2007). Propiedades psicométricas de la versión española del Inventario de Ansiedad Competitiva CSAI-2R en deportistas. Psicothema, 1, 150155.

Bandura, A. y Walter, R. (1983). Aprendizaje Social y Desarrollo de la Personalidad. Ed. Alianza, Séptima Edición. Madrid, España.

Cabal, H. V. (2018). Adaptación para Deportes de Montaña (CPRD-M) del Cuestionario" Características Psicológicas relacionadas con el Rendimiento Deportivo"(CPRD). Revista Iberoamericana de Diagnóstico y Evaluación-e Avaliação Psicológica, 2(47), 185-196.

Folkman, S., Lazarus, R.S., Dunkel-Schetter, Ch., DeLongis, A. y Gruen, R.J. (1986). Dynamics of a stressful encounter: Cognitive appraisal, coping, and encounters outcomes. Journal of Personality and Social Psychology, 50(5), 992-1003.

García, N. B. (2011). Estrés académico. Revista de psicología Universidad de Antioquia, $3(2), 55-82$.

García-Cerda, L., Rodríguez-Fernández, O., Betancourt-Galindo, R., Saldívar-Guerrero, R., \& Torres-Torres, M. (2003). Síntesis y propiedades de ferrofluidos de magnetita. Superficies y Vacio., 16(1), 28-31.

Garzón, BA. (2015). Incidencia del estado de Pre Arranque en el Rendimiento de los Jugadores de Futbol Sala del Club Deportivo AT\&T de la Provincia de Pichincha periodo Enero -Julio de 2014. Tesis aspirante al grado académico de Master en Ciencias.

González, G., Valdivia, P., Zagalas, M. (2015). La Autoconfianza y el Control del Estrés en Futbolistas: revisión de estudios Revista Iberoamericana de Psicología del Ejercicio y el Deporte, vol. 10, núm. 1, enero-junio, 2015, pp. 95-101 Universidad de Las Palmas de Gran Canaria Las Palmas de Gran Canaria, España

Gimeno, F., Buceta, J. M. y Pérez-Llantada, M. C. (2001). El cuestionario características psicológicas relacionadas con el rendimiento deportivo (CPRD): características psicométricas. Análise Psicológica, 1, 93-113. 
Mateos-Pérez, E. \&. (2019). Interacción entre diferentes factores cognitivos en la predicción de los síntomas de depresión. Psicología Conductual, 27(1), 21-39.

Mocha Bonilla Julio, J. R. (2018). EFFECTS OF MOTIVATION IN SPORTS: A STUDY WITH PEOPLE WITH VISUAL IMPAIRMENT. Ponte Academic Journal Multidisciplinary Journal of Science \& Research, 74(1), 16-24. doi:doi: 10.21506/j.ponte.2018.1.32

Molinero, O. S. (2012). Estrés-recuperación en deportistas y su relación con los estados de ánimo y las estrategias de afrontamiento. Revista de psicología del deporte, 2(1), 0163-170.

Moreno-Jiménez, B. G.-H.-H. (2005). Personalidad positiva y salud. Psicología de la salud. Temas actuales de investigación en Latinoamérica, 59-76.

Paz-Llanos, G. C.-H.-C. (2019). Características psicológicas del rendimiento deportivo y la motivación en deportistas de patinaje de velocidad. Cultura Física y Deportes de Guantánamo, 9(16).

Pedrosa García, I., Suárez Álvarez, J., \& García Cueto, E. (2012). Construcción de una Escala de valoración del Estrés en el Ámbito Deportivo (EEAD). REMA.

Piqueras, I., Casinello, P. (2011). Afrontamiento al Estrés y la Ansiedad en el Ámbito Deportivo. Recuperado 9 de febrero de 2019. En: https://www.efdeportes.com/efd152/estres-y-la-ansiedad-en-el-ambitodeportivo.htm. Revista Digital-Buenos Aires- Año 15 enero 2011.

Suntaxi, W. (2015). El Estrés en el Rendimiento Deportivo de la Categoría Senior de Combate del Club de Taekwondo de la Universidad de las Fuerzas Armadas ESPE (Sangolquí).

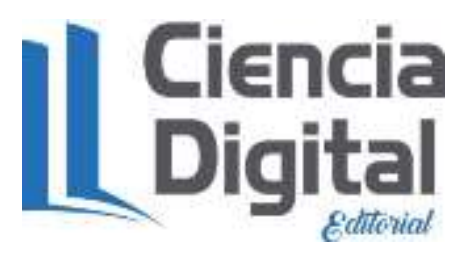




\section{PARA CITAR EL ARTÍCULO INDEXADO.}

Pérez Esquivel, V., \& Díaz Martín, Y. (2019). Intervención Psicológica para la Gestión del Estrés en Deportistas Juveniles Federados. Ciencia Digital, 3(2.5), 105-119. https://doi.org/10.33262/cienciadigital.v3i2.5.535
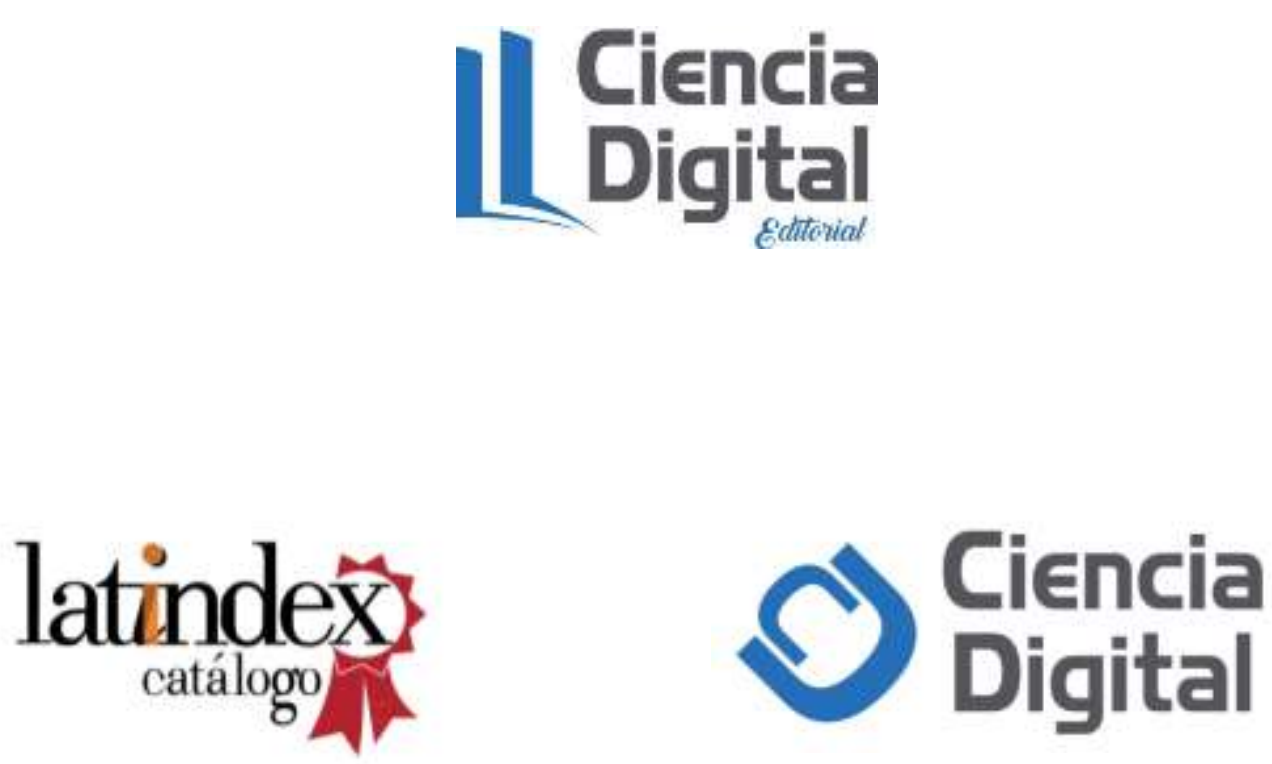